\title{
EL VALOR DE LA FILOSOFÍA PARA LA FORMACIÓN UNIVERSITARIA
}

\author{
MAURICIO ALBARRACÍN \\ Universidad de los Andes \\ JAVIER AGUIRRE ROMÁN \\ Universidad Industrial de Santander
}

(c) $\underset{\substack{1 \\ \text { ov }}}{\mathbb{2}}$ 


\title{
EL VALOR DE LA FILOSOFÍA PARA LA FORMACIÓN UNIVERSITARIA*
}

Resumen: el profesor Javier Aguirre Román, director de la Escuela de Filosofía, de la Universidad Industrial de Santander, entrevista a Mauricio Albarracín, profesor e investigador de la Universidad de los Andes y activista por los derechos de la comunidad LGBTI, en el marco de la II Semana de Investigación y Extensión "Filosofía y acción política" y el III Encuentro de Egresados de la Escuela de Filosofía de la UIS.

Palabras clave: derecho, filosofía, reflexión, universidad, formación.

\section{THE PHILOSOPHY VALUE FOR UNIVERSITY EDUCATION}

\begin{abstract}
The professor Javier Aguirre Román talked with Mauricio Albarracín, professor and researcher of Universidad de los Andes, and activist for rights of LGBTI community in the II Semana de Investigación y Extensión "Filosofía y acción política" and the III Encuentro de Egresados de la Escuela de Filosofía UIS. In this paper we share the conversation about the rol the Philosophy in the educational field.
\end{abstract}

Keywords: law, Philosophy, thought, university, education.

Forma de citar (APA): Aguirre, J. y Albarracín, M. (2016). El valor de la filosofía para la formación universitaria. Revista Filosofía UIS. 15 (1), 137-141, doi: http://dx.doi. org/10.18273/revfil.v15n1-2016006

Forma de citar (Harvard): Aguirre, J. y Albarracín, M. (2016). El valor de la filosofía para la formación universitaria. Revista Filosofía UIS. 15 (1), 137-141.

Fecha de recepción: octubre 28 de 2015

Fecha de aceptación: octubre 28 de 2015

Mauricio Albarracín: colombiano, filósofo y abogado egresado de la UIS. Magíster en Derecho de la Universidad de los Andes y de American University Washington College of Law. Fue director ejecutivo de Colombia Diversa y actualmente es investigador del Centro de Estudios de Derecho, Justicia y Sociedad-Dejusticia de la Universidad de los Andes. Es un reconocido activista por los derechos de la comunidad LGBTI.

Correo electrónico: malbarracin@gmail.com

Javier Aguirre Román: colombiano, profesor asociado y director de la Escuela de Filosofía UIS. Es Ph.D. en Filosofía por Stony Brook University. Como Mauricio, también es abogado y filósofo egresado de la Universidad Industrial de Santander.

Correo electrónico: javierorlandoaguirre@gmail.com

* Entrevista.

Revista Filosofía UIS, Vol. 15, No. 1, enero-junio de 2016 pp. 137-141 


\section{EL VALOR DE LA FILOSOFÍA PARA LA FORMACIÓN UNIVERSITARIA}

Javier Orlando Aguirre Román: estamos con Mauricio Albarracín Caballero, él es un egresado destacado de la Universidad Industrial de Santander, doblemente destacado, doblemente egresado; es filósofo y abogado. Posteriormente, Mauricio, si no estoy mal, hizo un magíster en la Universidad de los Andes y también otro máster en la American University Washington College of Law. Este último, en virtud de una beca Fulbrigth que obtuvo en el 2012. Además, fue director ejecutivo de Colombia Diversa. Actualmente, es investigador del centro de estudios de Derecho, Justicia y Sociedad, de la Universidad de los Andes. También, es un reconocido activista, defensor de los derechos de la comunidad LGBTI e incluso este año, fue elegido por la revista Semana como uno de los veinte mejores líderes de Colombia, llamado "el luchador de la igualdad".

Realmente hay muchas preguntas que te quisiéramos hacer (hoy estás invitado a la II Semana de Investigación y Extensión y el III Encuentro de Egresados de la Escuela de Filosofía; es un evento que tenemos y que nos complace mucho que nos acompañes, gracias a la oficina de Relaciones Exteriores); en primer lugar, no sé si te hayas topado con la pregunta de siempre que es: bueno, Filosofía... ¿Filosofía para qué?, ¿qué se hace con la Filosofía? Tú tienes formación de filósofo y abogado, por esto quisiera conversar contigo acerca de cómo y en qué te ha ayudado la formación filosófica, que para bien o para mal, tuviste y continúas teniendo.

Mauricio Albarracín: Yo empecé a estudiar Derecho porque de las ciencias humanas y sociales era la que auguraba una mejor salida profesional. Entonces, fui abogado más por la utilidad que parecía tener el Derecho. Luego, me pareció que estudiar Derecho era una carrera muy útil, pero intelectualmente muy pobre. Dado que siempre he tenido una tendencia a estudiar algo como sociología o antropología, pero la ciudad no ofrecía tantas opciones, terminé en Filosofía como por casualidad. Entonces, decidí estudiar simultáneamente Derecho y Filosofía. Sin embargo, aprendí más Filosofía que Derecho. Ahora, creo que uno va a la 
universidad a aprender a leer y escribir; en Filosofía aprendí esa pasión por leer ciertos autores a profundidad.

Estudiaba Derecho por la mañana y Filosofía por la tarde. Las clases de Derecho eran menos intelectuales; puesto que los profesores tenían cierta visión utilitarista del conocimiento, pues insistían: "Hay que aprenderse esto para tal cosa". Mientras que en Filosofía nada parecía tener una utilidad evidente para la vida. Así, terminé ambas carreras y soy profesionalmente más un abogado por las calidades profesionales, pero creo que el sustrato intelectual que da la Filosofía no lo da el Derecho.

Por esta razón, cuando tengo dudas no leo un texto legal, sino un texto filosófico, cuando escribo una columna semanal lo que me saca a veces de los líos intelectuales es la Filosofía. De esta manera, la filosofía se volvió para mí esa fuente de reflexión en mi vida.

Entonces, para concluir, puedo decir que empecé a estudiar filosofía como una curiosidad intelectual. Luego estudié un año en la Nacional. Y de ese estudio entendí que uno se siente mejor cuando estudia con amigos y no con competidores, porque estudiar con competidores hace que los procesos intelectuales se vuelvan cargas en la vida. Y este sentido de la amistad para el aprender, en vez de la competencia, lo viví acá en la carrera de Filosofía de la UIS y no en la de la Nacional.

J.O.A.R.: Ahora bien, desde que te conocí siempre has sido una persona bastante crítica, pero a la vez generosa con tus reflexiones. Seguramente, hay muchas cosas que podríamos hacer mejor. Por eso no quisiera desaprovechar esta oportunidad para preguntarte: ¿qué podría mejorar la formación filosófica para que sea más rica, más interesante?

M.A.: Tres cosas: cuando estudié Filosofía vi mucho 'disciplinarismo'; es decir, la ansiedad de ser una disciplina distinta de otras. El segundo problema es que le han dejado el espacio de la intervención intelectual pública a gente mediocre. Las universidades públicas perdieron su vocación de liderazgo. Y un tercer problema con las escuelas de filosofía es la invisibilidad. Allí se hacen trabajos muy interesantes, pero ocurre que nosotros no valoramos lo que tenemos. La gente que estudia filosofía (aunque esto también aplica para otras carreras, sobre todo en la universidad pública) tiene que creer que lo que hace es valioso, importante y puede tener capacidad de impacto.

J.O.A.R.: Para terminar, quisiera que nos comentaras un poco acerca de tus apreciaciones sobre el proceso de paz y el posconflicto. Entiendo que parte de tu activismo político te ha llevado a relacionarte de forma directa con las negociaciones de la Habana. 
M.A.: El nuestro es un conflicto de baja intensidad pero de larga duración, que ha carcomido todos los estamentos de la sociedad. Pero la mayor empresa del país hoy es la paz. Yo creo que Colombia es capaz de reconstruirse; Colombia ha tenido unas 12 guerras civiles y ha tenido 12 reconstrucciones. Las universidades tienen el privilegio de tener un espacio para leer, para pensar, para estudiar, en donde se inspiren las nuevas generaciones y se impulse un cambio a través de lo académico. Este es un momento para pensar, para crear. Y eso es lo que puede permitir la paz: poder imaginarse una situación en la cual uno puede, a través de las ideas y del deseo de cambiar la realidad. Y este debe ser uno de los propósitos más importantes del quehacer universitario.

J.O.A.R.: Mauricio, muchas gracias por tu visita y tu participación en nuestros eventos. También quiero agradecer al profesor Viatcheslav Kafarov, director de Relaciones Exteriores, y a la profesional Johanna Delgado, por posibilitar la presencia Mauricio en estas jornadas de la Escuela de Filosofía $\varphi$

\section{REFERENCIAS}

Gimeno, J. (Comp.) (2010). Saberes e incertidumbres sobre el currículum. Madrid: Morata.

Organización de las Naciones Unidas para la Educación, la Ciencia y la Cultura (2011). La filosofía, una escuela de la libertad-Enseñanza de la filosofía y aprendizaje del filosofar: la situación actual y las perspectivas para el futuro. México: UNESCOUniversidad Autónoma Metropolitana, Unidad Iztapalapa. 